\title{
Cancer stem cells on demand
}

Expressing four factors in glioblastoma cells can induce a stem cell state, a technique that can improve our understanding of the disease and yield new therapeutic targets.

Stem cells are engines that drive development, producing progenitors that differentiate progressively into the various tissues of the adult. In some cancers, stem-like cells may use a similar process to maintain tumor growth. To understand the structure of the disease and to head off long-term resistance to therapy, Mario Suvà and Bradley Bernstein of Harvard Medical School and their colleagues found a way to identify and generate stem-like cells of the incurable, aggressive brain cancer glioblastoma.

Starting with primary human tumors, the researchers cultured cells under two conditions: with serum, to produce cells with some differentiated properties, and without serum, to produce stem-like tumor promoting cells
(TPCs). Each pair of cultured cells contained essentially identical genomic sequences, but whereas an injection in mice of just 50 TPCs could form tumors, 100,000 serum-cultured glioblastoma cells formed none.

In search of what distinguished the cell types, the researchers sequenced RNA and profiled histone $\mathrm{H} 3$ lysine 27 acetylation, an epigenetic mark of active enhancers, for each sample pair. They found 19 transcription factors with higher RNA levels in TPCs that also bound DNA sequences overrepresented in TPC-specific enhancers.

To test whether any of these could direct cancer stem cell fates, the team expressed each factor individually and in combinations in differentiated glioblastoma cells. Extensive testing revealed a specific set of four transcription factors that produce reprogrammed cells capable of forming tumors in mice and that share epigenetic and expression profiles with TPCs.
The factors provide a molecular signature for studying TPCs in vivo as well as regulatory pathways that can be targeted for intervention. Chromatin immunoprecipitation identified potential genomic binding sites of the four factors. One common regulatory target is a co-repressor that functions with the histone methyltransferase lysine-specific demethylase 1 (LSD1). Reducing the activity of LSD1 genetically or with an inhibitor profoundly dropped TPC viability, suggesting a promising therapy.

The ability to induce cancer stem cells provides a needed tool to study and target the small cell population that drives cancer growth and long-term resistance to medical treatment.

Tal Nawy

\section{RESEARCH PAPERS}

Suvà, M.L. et al. Reconstructing and reprogramming the tumor-propagating potential of glioblastoma stem-like cells. Cell 157, 580-594 (2014). 